\title{
Non-rigid Multimodal Image Registration Using Mutual Information
}

\author{
Tom Gaens, Frederik Maes, Dirk Vandermeulen, Paul Suetens \\ Laboratory for Medical Imaging Computing, ESAT/Radiology, Univ. Hosp. \\ Gasthuisberg, Katholieke Universiteit Leuven, Herestraat 49, B3000 Leuven, Belgium \\ e-mail: Tom. Gaens@uz.kuleuven.ac.be
}

\begin{abstract}
A fully automated voxel-based algorithm for multimodal non-rigid image registration using a mutual information based similarity criterion is presented. The image deformation vector field is found by dividing images into overlapping neighborhood regions and translating these regions to locally increase the similarity criterion. The calculated displacement in each region is assigned to the centered pixel of each neighborhood region and propagated to its neighbors with a Gaussian window function assuring continuity and smoothness of the deformation field. Initial experiments on 2D test data qualitatively demonstrate the feasibility of this approach.
\end{abstract}

\section{Introduction}

Images from different medical imaging modalities often provide complementary information to the clinician, and therefore image alignment or registration has found several applications in diagnosis, treatment planning, and therapy followup. Registration algorithms (see [18] [20] for an overview) can be classified as being either stereotactic frame based, landmark based, surface based, or voxel based. Voxel-based methods optimize a metric measuring the similarity of all geometrically corresponding voxel pairs for some feature. Until recently, research was mainly focused on the search for an adequate voxel-based similarity measure as an optimization functional. Proposals for new and better alignment measures culminated in the use of information-theoretic matching criterions. An example of this approach is the concept of mutual information, which was introduced independently by Collignon et al. [8] and Viola and Wells [21].

Traditional registration methods apply global and rigid transformations, which is useful in cases where rigid body assumptions are likely to hold. However, they cannot account for non-rigid morphological variability between and within subjects. For applications where this variability matters, e.g. segmentation of brain structures by matching an individual brain to a labeled brain template, non-rigid matching must be used. Other applications include registration of intra-operative imaging data with pre-operative images that delivers intra-operative guidance.

In non-rigid matching, a deformation vector is determined for each image voxel in such a way that, applying the total deformation vector field, causes the 
similarity criterion to reach an optimal value and both images to get geometrically aligned. The vast amount of degrees of freedom introduced in non-rigid matching can be reduced by imposing constraints on the deformation field.

Static constraints are imposed via landmark or control points. The deformation in a certain point is then obtained by interpolation of the known deformations in the landmark points (e.g. by calculating a spline through the landmarks [2]). Succesful work on using mutual information as a similarity measure and thin-plate spline algorithms as static constraints on the deformation field are found in [15] for intra-operative guidance, and more generally in [16]. However, the corresponding control points need to be found manually or semiautomatically, and their limited number is too restrictive.

This problem can be alleviated by formulating registration as a dynamics problem, where dynamic constraints are imposed on the deformation field by modeling the imaged objects to be aligned as deformable solid bodies. External forces trying to deform objects are gradients of a similarity criterion, causing the similarity to increase. The deformation is constrained by internal forces generated because of the physical properties of the object. Early work using NavierLamé equations found in elasticity theory was reported by Bajcsy et al. [1], based on work by Burr [6] and Broit [3]. Plastic registration [10], has even more degrees of freedom than elastic registration. Very little constraints are imposed on the possible deformations, except that topology must be preserved and that the transformation must be continuous and smooth. Plastic registration can be thought of as deforming a viscous fluid. The more viscous the fluid is, the more it will resist deformation and consequently the more smooth the transformation will be. Because of this kind of interpretation, plastic registration algorithms have also been called Fluid Transforms in the literature. Christensen [7] therefore uses Navier-Stokes equations found in hydrodyamics theory to regularize the computed deformation field. In developing a speeded-up implementation of the fluid approach of [7] Bro-Nielsen [4] noticed the correspondence between these Tikhonov-type of regularization methods and the Gaussian scale space. Note that Thirion's [19] so-called Maxwellian 'demon'-based method in [19] makes effectively use of this correspondence. In a practical and approximative implementation, the problem of regularization boils down to the choice between Gaussian smoothing of either the total calculated deformation field (i.e. elastic registration) or the incremental deformation field (i.e. fluid registration), since the Gaussian function is the Green's function of the heat or diffusion equation. In this case, the regularizer is the diffusion equation, that propagates deformations to neighboring points [12]. Hence, one can say that the demons method is a trade-off between speed of calculations and accuracy of the results.

Most of the above mentioned non-rigid matching methods use a least squarederror distance measure as a similarity metric (the so-called Gaussian sensor model). As a lot of clinical applications ask for inter-individual and multi-modal matching, several more effective matching criteria were proposed (e.g. correlation measures [10], or feature-based potentials [7]). Recently, mutual information has been used as a similarity criterion in a dynamics based method, presented by 
Maintz et al. [17]. The main drawback of their method is the implicit assumption that probabilities computed after rigid registration are good approximations of the same probabilities after fluid matching.

In this paper, we propose a dynamics based registration algorithm [12] using mutual information, and without these limitations. It's a variant of the uni-modal image registration method explored by D. Collins [10], where image transformations are found by dividing images into regions and translating these regions to increase the local similarity criterium. The theoretical aspects of our algorithm and implementation issues are described in section 2. In section 3 , some results on artificial and real images are presented, followed by a discussion in section 4 .

\section{Algorithm}

\subsection{Similarity metric}

The information-theoretic measure of mutual information [11] measures the amount of information one random variable contains about another random variable. The mutual information of two images is maximal when they are geometrically aligned (see [9] for a rigorous discussion). The advantage of this criterion is its generality, since it does not assume a certain relationship between the grey-values in the images to be registered [14].

Let $A$ and $B$ be two images with marginal entropies $H(A)$ and $H(B)$, and joint entropy $H(A, B)$ then the expression of mutual information can be written as: $I(A, B)=H(A)+H(B)-H(A, B)$. Marginal entropies $H(A)$ and joint entropy $H(A, B)$ are defined using joint and marginal probability distributions, which are in turn estimated using image histograms (see [14] for definitions).

\subsection{Outline}

The goal of our method is to calculate a deformation field that maximizes the mutual information functional. Calculating a displacement separately for each image voxel, makes the problem ill-posed. Because of the statistical nature of mutual information, centered neighboorhood regions are defined in each point of a discrete lattice $\left\{x_{i} \mid i=1, \ldots, N\right\}$, of the image to be registered ( $N$ is the number of dimensions). This image is then locally transformed by translating every neighborhood region so that the total mutual information increases. Therefore the incremental changes to the joint histogram and marginal histograms are computed.

The calculated displacement in $x_{i}$ is then propagated to each point in the neighborhood region by a propagation function, which in practice can be a Gaussian kernel. This deformation propagation can be interpreted in 3 ways. The most direct interpretation is to consider the image as a viscous fluid and the deformation propagation as the viscosity of this fluid. Alternatively one can interpret the Gaussian as a low pass filter and deformation propagation as convolving the deformation field with this low pass filter. Finally one can interpret the Gaussian as a radial basis function and the deformation propagation as a radial basis 
function spline expansion of the deformation field. Note that the deformation propagation ensures continuity and smoothness of the deformation field.

Because the deformations of neighbouring nodes are not independent, the deformation in every node cannot be estimated in one pass, so this process is repeated iteratively. The iterative process is stopped when the incremental change to mutual information falls below a threshold.

As topology must be preserved, $D\left(x_{i}\right)$ may not 'jump' over any of its transformed neighboring nodes: this is accomplished by appropiately rescaling the incremental updates of the deformation field (for a more rigorous treatment of topology violation, see [13]).

\subsection{Implementation issues}

In order to speed up the algorithm, some approximations were made to the above outlined method. Sub-voxel translations were not considered in moving the neighborhood region, to avoid additional interpolations. Another change was to consider translations only in the direction of the image gradient of the floating image, hereby assuming that iso-intensity curves deform only in the direction of the normal onto these curves. Note the equivalence with the optical flow based demons method described in [19], and the fact that the number of evaluations of the criterion is hereby reduced.

By translating the neighorhood region around each image voxel, local transformations are found, that are propagated into a continuous and smooth transformation. Because macroscopic features are generally more stable than microscopic ones (for human anatomy), it would be useful to recover first more global deformations and in a later stage fine and detailed deformations. This can be controlled in three ways: by using multi-resolution pyramids, by varying the neighborhood region size, and by varying the number of bins in calculating histograms.

Using multi-resolution image pyramids, calculation proceeds from coarse to fine, i.e. from the images with the lowest resolution to the highest resolution. Because of the fact that, by filtering the image the cost function is also smoothed (i.e local minima are filtered away) faster convergence to a global optimal value will be made easier. In practice only few levels suffice. The size of the neighborhood region can also be varied on a large-to-small basis. In doing so, large deformations are propagated at low resolution levels, and fine and detailed deformations are recoverd at high resolution levels. As binning corresponds to a raw labeling of the imaged objects, the number of bins to build up the histograms can be chosen on a small-to-large basis. In this way, one considers large 'objects' at the coarsest resolution levels and small detailed 'objects' at the finest resolution levels.

\section{Results}

\subsection{Test on Artificial Multi-Modal Images}

To evaluate the registration algorithm the image grid of an MR slice (size $256 \mathrm{x}$ 256 pixels) was deformed using an analytically known transformation. In order 
to simulate multi-modality, the intensities of this deformed image were changed with a sine function: $\sin (\pi *$ intensity $)$. In this way, the reference image was created artificially, depicting a similar object imaged with a different modality, with a non-linear and one-to-many relationship between the image intensities of floating and reference images.

To be able to compare the images quantitatively, the theoretical deformation field was applied to the original MR slice, hereby creating a uni-modal equivalent to the reference image. Test setup and results are depicted in figure 1.

\subsection{Test on Real Multi-Modal Images}

Tests were also carried out on real multi-modal images. Therefore a pair of proton-density and T2 images was used, because they are already optimally registered at acquisition. The T2 image was taken as floating image, while the proton-density image was deformed using a similar exponential deformation field as used for the artificial images.

The comparison of the result with the theoretical solution was possible because of the fact that the original PD and T2 images were aligned from the start, and was made in the same way as with the artificial images: the theoretical deformation field was applied to the original T2 slice, hereby creating a uni-modal equivalent to the reference PD image. Test setup and results are depicted in figure 2 .

\subsection{Application: Atlas-driven Segmentation}

One could use non-rigid registration to match images to a digital anatomical atlas for the segmentation of brain (sub)structures. In the ideal case, this could be accomplished automatically by an adequate registration algorithm, that brings images into correspondence within the same reference frame of the atlas.

As a test case for our algorithm, we made a segmentation map of a set of $\mathrm{T} 2$ and proton-density images of one patient A. The image created in this way was considered the 'atlas'. This map was then registered non-rigidly to a T2 image of another patient B (together with a corresponding proton-density image, considered 'study' images). Evaluation of the result is then possible by comparing the deformed 'atlas' to the segmentation of the 'study' images of patient B. Qualitative results are shown in figure 3 . Notice that the result could still improve by registering the segmentation map to both study images (this requires building a 3D joint histogram from the atlas and both $\mathrm{T} 2$ and $\mathrm{PD}$ study images).

\section{Discussion and Conclusion}

In this work, a non-rigid registration algorithm using an information-theoretic criterion has been presented. The image transformation was found by dividing the image into small regions and translating these regions to increase the local 

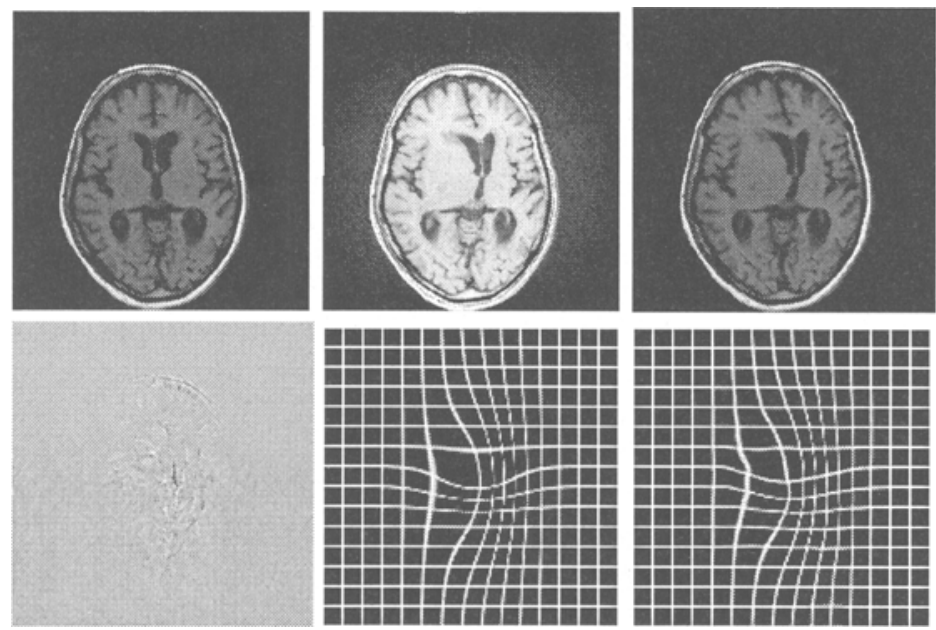

Fig. 1. Test setup and results for artificial images; from top left to bottom right: floating image, reference image, deformed floating image, difference between calculated deformed floating image and theoretically deformed floating image (intensities rescaled to $[0,1]$ ), theoretical grid deformation (subsampled detail), calculated grid deformation (subsampled detail)
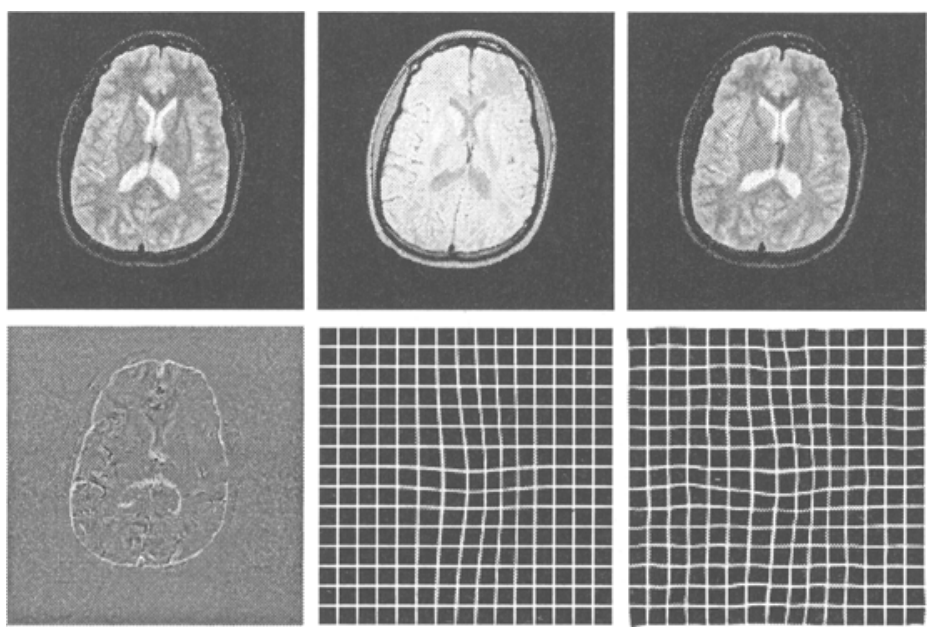

Fig. 2. Test setup and results for real images; from top left to bottom right: T2 floating image, PD reference image, deformed floating image, difference image between calculated T2 image and theoretically deformed T2 image (intensities rescaled to $[0,1]$ ), theoretical grid deformation field (subsampled detail), calculated grid deformation(subsampled detail) 

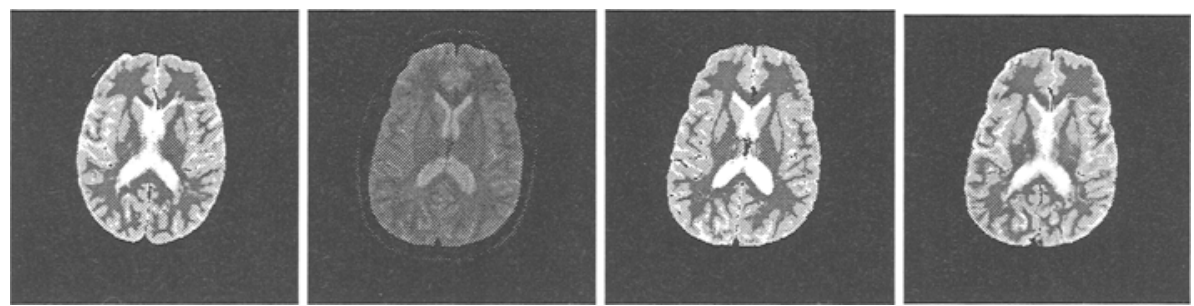

Fig. 3. Preliminary result for atlas segmentation; from left to right: segmentation map of patient A, i.e. the 'atlas' (floating image), T2 reference image of patient B, segmentation map calculated from $\mathrm{T} 2$ reference image and corresponding $\mathrm{PD}$ image of patient $\mathrm{B}$, deformed floating image

similarity criterium. The floating image was considered a viscous fluid and the deformation propagation the viscosity of this fluid.

Because the resulting deformation field is smooth and continuous, the algorithm cannot account for topological differences (as can be seen in the results of figure 3.

One has to bear in mind that the 'physical' descriptions of elastic and fluid registration model the whole image grid as one physical object, and not the separate objects seen in the image. Modelling the different imaged objects as separate tissues would require an a priori segmentation, and a physically correct model for each of the segmented objects depending on the application (for intra- and inter-individual morphometric studies, e.g. based on physical growth models [5] or morphogenesis). It also implies the modeling and calculation of discontinuous and non-smooth deformation fields for applications in intra-operative guidance.

\section{Acknowledgements}

This research was performed within the context of the EASI project "European Applications for Surgical Interventions", supported by the European Commission under contract HC1012 in their "4th Framework Telematics Applications for Health" RTD programme, and using research grant KV/E/197 (Elastische Beeldregistratie voor Medische Toepassingen) of the FWO-Vlaanderen to Dirk Vandermeulen, research grant COF 96/12 - AO655 (Actie van de Vlaamse Minister van Wetenschapsbeleid ter stimulering van de Vlaamse Deelname aan EUprogramma's), and research grant G-0340-98 of the FWO-Vlaanderen.

\section{References}

1. R. Bajcsy and S. Kovacic, Multiresolution elastic matching, Computer Vision, Graphics, and Image Processing, 46:1-21, Academic Press, 1989.

2. F.L. Bookstein, Morphometric tools for landmark data. Geometry and biology, Cambridge University Press, 1991. 
3. C. Broit, Optimal registration of deformed images, PhD thesis, University of Pennsylvania, August 1981

4. M. Bro-Nielsen and C. Gramkow, Fast fluid registration of medical images, in Proc. Visualization in Biomedical Computing (VBC'96), Springer Lecture Notes in Computer Science, 1131:267-276, September 1996.

5. M. Bro-Nielsen, C. Gramkow, and S. Kreiborg, Non-rigid image registration using bone growth model, in Proc. CVRMed/MRCAS'97, Springer Lecture Notes in Computer Science, 1205:3-12, 1997.

6. D.J. Burr, Elastic Matching of Line Drawings, IEEE Trans. on PAMI, 3(6):708713, November 1981

7. G.E. Christensen, Deformable shape models for anatomy, PhD thesis, Washington University, Sever Institute of Technology, 1994.

8. A. Collignon, F. Maes, D. Delaere, D. Vandermeulen, P. Suetens, and G. Marchal, Automated multimodality medical image registration using information theory, in Y. Bizais, C. Barillot, and R. Di Paola (Eds.), Proc. XIV'th Int. Conf. Information Processing in Medical Imaging, Computational Imaging and Vision 3, pp. 263-274, Kluwer Academic Publishers, June 1995.

9. A. Collignon, Multi-modality medical image registration by maximization of mutual information, $P h D$ thesis, K.U. Leuven, 1998

10. D.L. Collins, 3D Model-based segmentation of individual brain structures from magnetic resonance imaging data, $P h D$ thesis, McGill University, 1994.

11. T.M. Cover and J.A. Thomas, Elements of Information Theory, John Wiley \& Sons, 1991.

12. T. Gaens, S. Sagaert, D. Vandermeulen, Non-rigid registration using mutual information, ESAT/PSI Technical Report nr. 97/10, 1997.

13. H. Lester, S. Arridge, Summarising fluid registration by thin-plate spline warps with many landmarks, in Proceedings of Medical Image Understanding and Analysis 1997, Oxford, July 1997.

14. F. Maes, A. Collignon, D. Vandermeulen, G. Marchal, and P. Suetens, MultiModality Image Registration by Maximization of Mutual Information, IEEE Trans. on Medical Imaging, 16(2):187-198, April 1997.

15. D. McGarry, T. Jackson, M. Plantec, N. Kassell, J.H. Downs, Registration of functional magnetic resonance imagery using mutual information, Proc. SPIE Medical Imaging 1997, pp. 621-630.

16. C. Meyer, J. Boes, B. Kim, P. Bland, K. Zasadny, P. Kison, K. Koral, K. Frey, R. Wahl, Demonstration of accuracy and clinical versatility of mutual information for automatic multimodality image fusion using affine and thin-plate spline warped geometric deformations, Medical Image Analysis, 1(3):195-206, April 1997.

17. J.B. Maintz, E. Meijering, M. Viergever, General multimodal elastic registration based on mutual information, to appear in Proc. SPIE Med. Imaging, 1998.

18. C. Maurer, and J. Fitzpatrick, A review of medical image registration, in R. Maciunas (Ed.), Interactive Image-guided Neurosurgery, pp. 17-44, American Association of Neurological Surgeons, 1993.

19. J.-P. Thirion, Fast non-rigid matching of 3D medical images, INRIA Technical Report, nr. 2547, May 1995.

20. P. van den Elsen, E.-J. Pol, and M. Viergever, Medical image matching - a review with classification, IEEE Eng. in Medicine and Biology, pp. 26-38, March 1993.

21. P. Viola and W.M. et al. Wells III, Multi-modal volume registration by maximization of mutual information, Medical Image Analysis, 1(1):35-51, March 1996. 